\title{
Problems of Analyzing Microstructure Images in Assessing the Impact of Technological Parameters of Combined Strain Wave Hardening on the Quality of the Surface Layer
}

\author{
A.V. Kirichek ${ }^{1}$, D.L. Solovyev ${ }^{2}$, A.V. Khandozhko ${ }^{1}$, S.O. Fedonina ${ }^{1}$ \\ avkbgtu@gmail.com|murstin@yandex.ru|chandosh@yandex.ru|fedonina.sv2015@gmail.com \\ ${ }^{1}$ Bryansk State Technical University, Bryansk, Russia; \\ ${ }^{2}$ Vladimir State University, Murom branch, Murom, Russia
}

\begin{abstract}
The problems of analyzing metallographic images and the method of their solution using modern software for the analysis of metallographic images are described. There is given an analysis of microstructure images as the main indicator of the surface layer quality by the example of studying the research results of strain wave hardening combinations and chemical-thermal treatment, in particular the influence of previous strain wave hardening and subsequent thermal and chemical-thermal treatment on the alloy steel microstructure or previous thermal and chemical- thermal treatment and subsequent strain wave hardening. On the basis of the analysis the effectiveness of strain wave hardening and chemical and thermal treatment is established
\end{abstract}

Keywords: analysis, image, hardening, surface plastic deformation, surface layer, carburization, chemical and thermal treatment, microstructure

\section{Introduction}

Constant development of computer technologies and methods of digital processing of images allows to accelerate and simplify research in all fields of science and technology. Using image analysis in assessing the quality of the surface layer gives the opportunity to identify the best processing methods that would best meet the requirements of the surface layer.

The study of microstructure is one of the main tasks of materials science, which allows not only to vary the mechanical properties of the surface layer and the performance properties of the finished part with a change in the phase composition, but also to create innovative materials or improve the properties of existing materials. The effectiveness of the metallographic analysis depends on many factors, ranging from the quality of preparation of samples to the subjectivity of observations and low speed of the research process [1].

\section{Main Part}

Images of microstructure are a combination of various structural components with the most common geometric dimensions and shapes, distributed unevenly and differently oriented. The combination of these structural components often gives a complex result, which is difficult to interpret without a sufficient level of training. Therefore, the main requirement for the qualitative analysis of images is to select phase components on the microstructure image under study, followed by classification and analysis according to the most significant quantitative characteristics. These can be both geometrical parameters of grains and percentage ratio of structural components in the investigated image or on the desired depth of a sample. When studying not one image, but several linked images, it is possible to obtain complete information about the change in the phase composition of the microstructure at the sample depth concerned, for example, when studying the hardening of the surface layer. In this case, at the depth depending on the type of applied finishing and strengthening treatment (FST), structural components should vary either in size and orientation or phase components of the microstructure, and in the case of combined types of processing - both in size and phase components. Taking into account FST peculiarities, the hardened layer in most cases has an implicit boundary, the detection of which depends not only on the quality of preparing the microsection and the correct selection of pickling solution, but also on the physiological data of the researcher. When conducting a simpler study that is comparing several images of microstructures, there are not only the problems mentioned above, but also the problem of obtaining the original image. Even with completely identical preparation and processing of microsections, the final images of microstructures may differ in brightness and color rendering, which significantly complicates the processing and analysis of the data obtained by the researcher. Thus, the main tasks of implementation of microstructure studies, are segmentation, filtering of defects and selection of objects from the background, determining the limits of objects, as well as image recognition $[1,4]$.

When conducting research, especially on metallographic equipment, which does not allow to change such object characteristics as intensifying the image sharpness and brightness, segmentation is quite problematic. Special software is required to improve the image quality, allowing to select all structural objects [5, 8-10]. However, this does not solve all the problems of metallography. Even with a very long and high-quality preparation of samples micro-scratches may remain on the surface of the microsection, for example, when studying the modes of applying a softer and more plastic material (bronze) on a steel part. When processing the resulting image, the program may evaluate such defects incorrectly, which will negatively affect the final analysis of the microstructure. Therefore, when using auxiliary programs for research, it is still impossible to rely on the software fully [6] and there should be an ability for the operator to change and adjust the data during running of the program.

One of the most promising ways to solve these problems is to use auxiliary software that analyzes the images in order to increase the efficiency of quantitative analysis.

At present there are a sufficient number of programs for speeding up and simplifying the research process. The most 
effective are considered the following: PHOTOM, OPTIMUS, VIDEOTEST, IMAGE EXPERT PRO, IMAGE, AVISO, SMARTEYE and many others. These programs have all necessary algorithms for processing technical images: high-frequency and low-frequency filtering, selection of image boundaries, arithmetic and logical operations, brightness/contrast correction, etc. Image processing in this case is not aimed at improving visual perception, but at preparing it for further analysis [1,7,11,12].

The result of metallographic studies using specialized software is the statistical analysis obtained in the process of measuring the characteristics of objects and determining the mean of these values, as well as the construction of graphical dependencies for visualization of the analysis process. However, it is not possible to rely entirely on the results obtained by the software without further analysis of the data obtained in terms of evaluation of materials science.

The problems of analyzing microstructure images in assessing the quality of the hardened surface layer of parts is shown by the example of studies on hardening of alloy steel 10XSND. The study is carried out on a metallographic microscope to determine the phase composition of the hardened metal, the characteristic grain size, grain density, depth of hardening, as well as the detection of defects in the hardened surface layer. The study objective is to identify the most optimal combination of hardening of the part surface layer.

Samples identical in size and thickness were gradually subjected to different FST types. In the first case, the surface layer of parts was processed by surface plastic deformation - strain wave hardening (SVH) [2], and then subjected to chemical-thermal treatment (CTT), and in the second case previously hardened by CTT surface layer was then strengthened by wave deformation. CTT hardening was carried out in several stages: 1 . double-ended carburization; 2 . interrupted quenching: I-quenching at temperature of $930^{\circ}$, II-quenching at temperature of $790^{\circ}$, with cooling in oil; 3 . backing. Depending on the combination, surface plastic deformation hardening was carried out before or after CTT [3].

The samples under study were compared according to the microstructure of the hardened layer, Figure 1. The images obtained well characterize the problems of metallographic studies described above: different color rendition of images, identification of structural components and boundaries of the hardened layer. These images do not allow to define reliably the ratio value of structural components and their distribution over the entire depth of hardening. Taking into account the complex combined processing of parts it is problematic to determine the depth range of hardening with great accuracy, as there is no significant difference between the grain size and the change of phase composition. In this case, the hardening boundary smoothly passes into the microstructure of the sample core. Images were processed without using auxiliary software for analysis and preparation of images, which complicated the process of comparison and analysis of microstructures. All these measurements were carried out using a metallographic microscope.
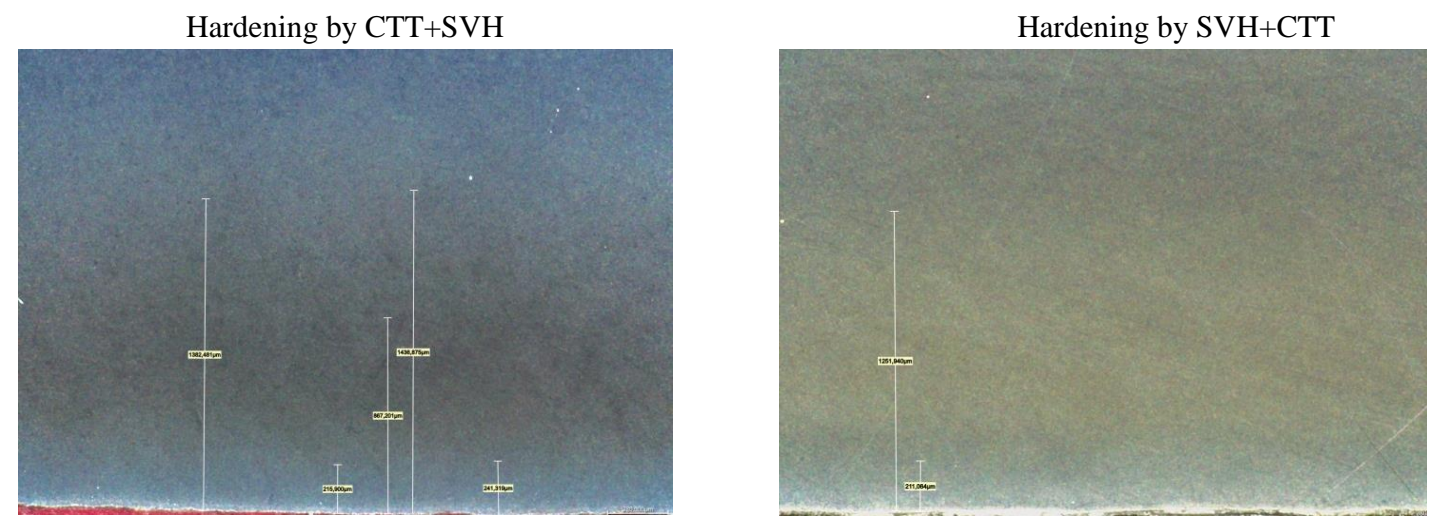

Depth of the hardened layer, $\mathrm{x} 158$
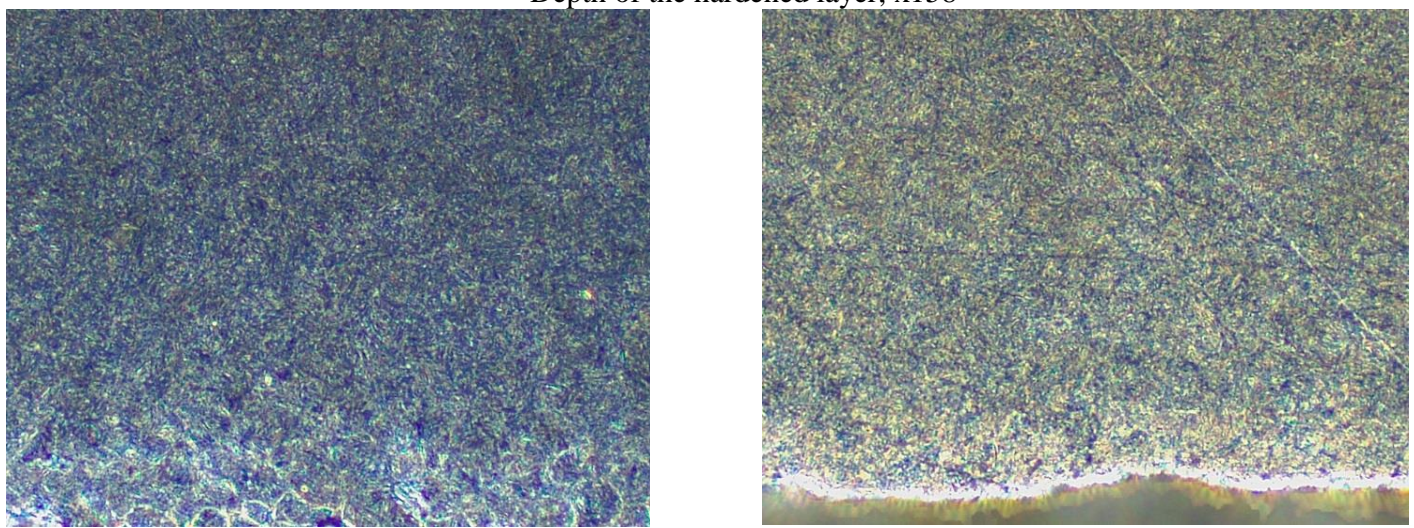

Subsurface layer of samples 

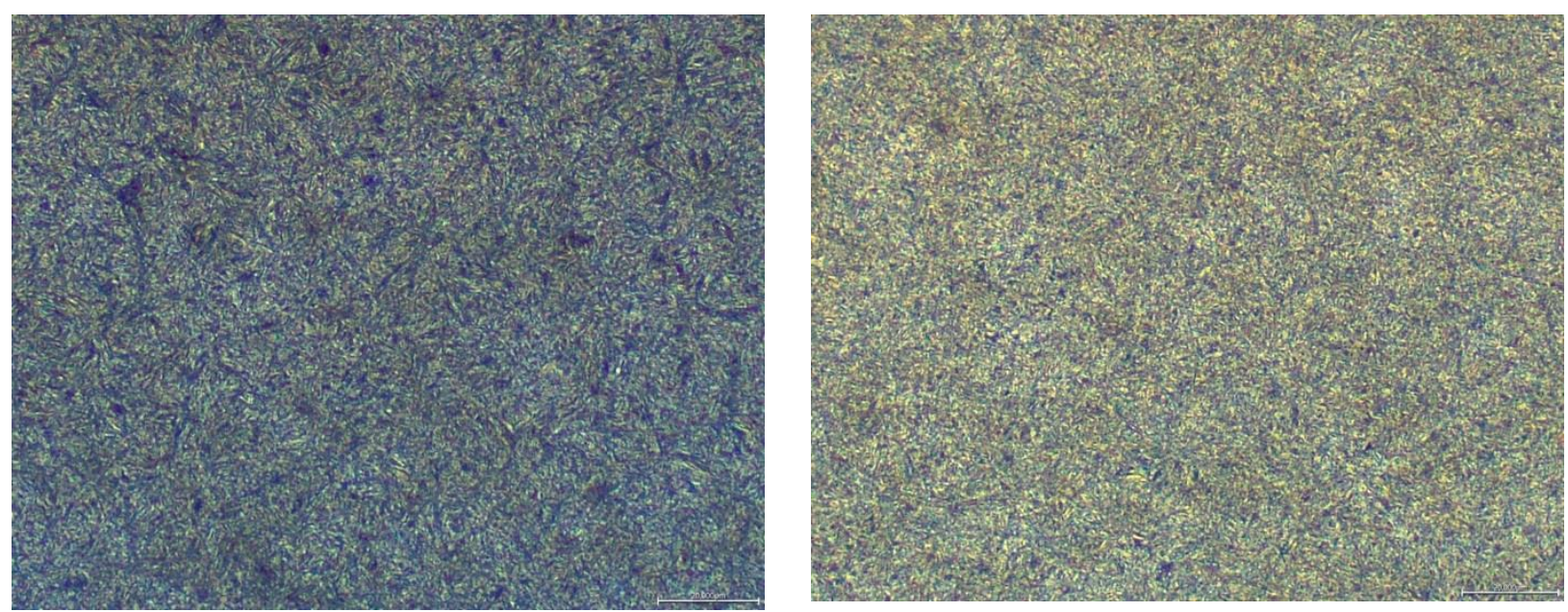

Depth $220 \ldots 320$ microns
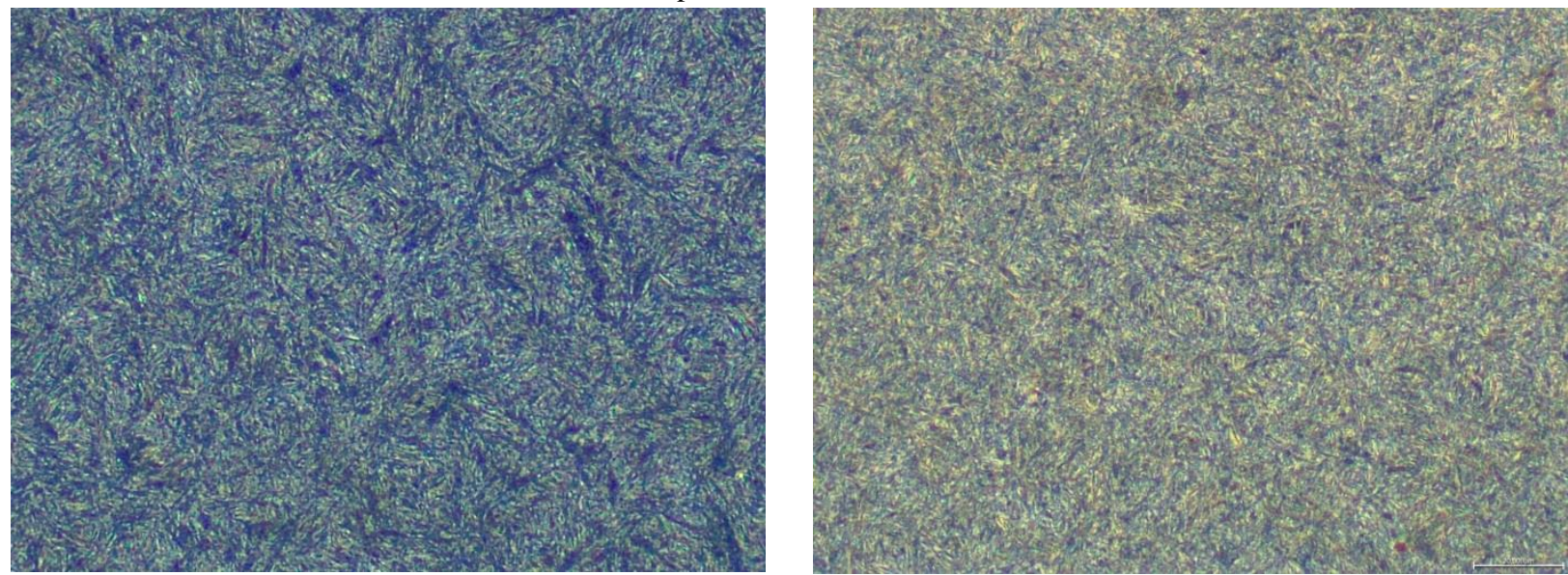

Depth $1400 \ldots 1500$ microns
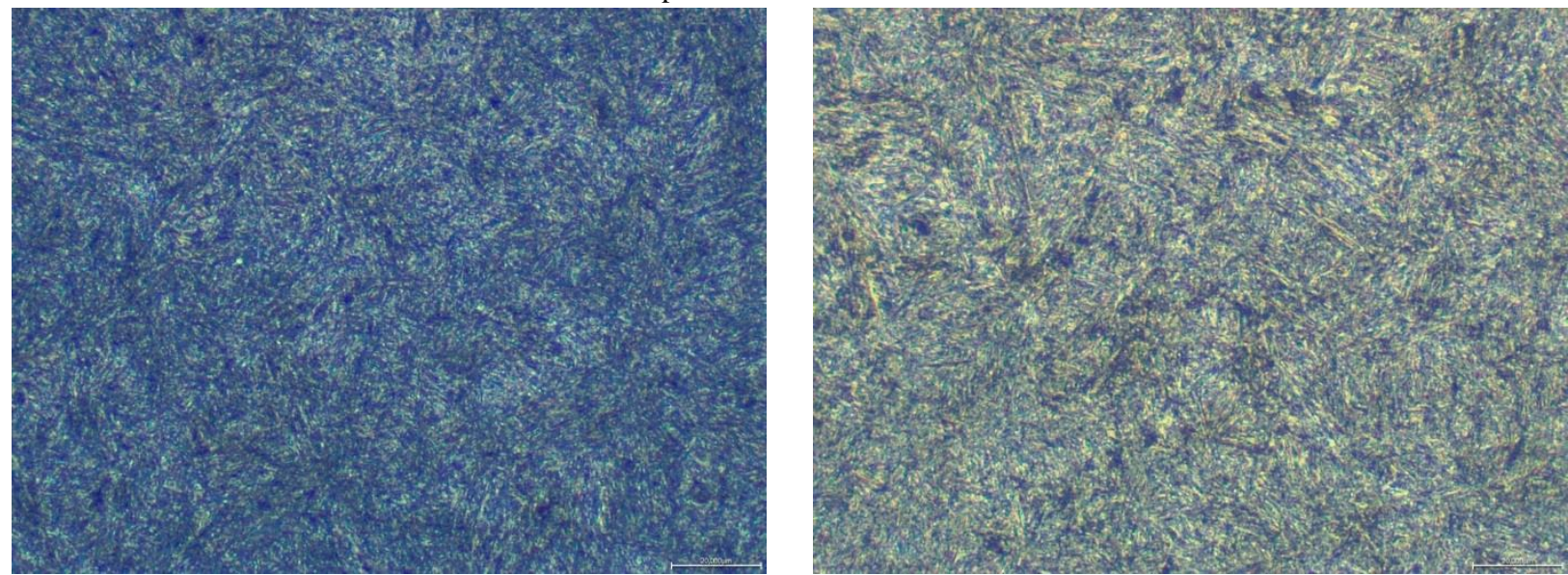

Sample cores

Fig. 1. Comparison of microstructures of 10KHSND steel samples hardened according to various schemes, $\mathrm{x} 2550$

Processing, analysis and assessment of the samples revealed the main features of microstructures. The microstructure of the subsurface layer of the sample, hardened according to $\mathrm{CTT}+\mathrm{SVH}$ type, is finely dispersed, but the grains are elongated of martensite. tempering. At the depth of about 220 microns, the combined structure begins: fine-needled martensite appears more clearly, the needles are up to 3 microns, there are a few small implicit bands of sorbite. At the depth of 400 microns, the microstructure is needlelike, there is a slight increase in the size of the needles up to 4-5 microns, there is a large number of sorbite bands. At the depth of 1400 microns, the microstructure is combined of three components - fine-needled, densely-packed martensite, fine grains and sorbite bands. No obvious martensite needles were found deeper than 
$1500 \ldots 1600$ microns, the microstructure gradually passes into the structure of the sample core.

The microstructure of the subsurface layer, hardened according according to SVH+CTT type, is finely dispersed. Fine-needled martensite appears at the depth of about 300 microns, the size of the needles is not more than 2 microns. This structure remains to the depth of $800 \ldots 850$ microns, after that it becomes denser, there are no clear martensite needles. At the depth of about 1500 microns, the grains are slightly elongated, densely-packed, even. Deeper the structure smoothly passes into the structure of the sample core, there is no explicit boundary of the hardened layer.

So CTT application to the previously hardened surface layer by wave deformation allows to form a finely dispersed structure to a greater depth and to create a smooth transition from the hardened zone to the non-hardened core of the sample. Due to the deformation effect on the loaded surface grains in the subsurface layer are crushed, which makes it possible to create a greater number of crystallization centers.

The use of auxiliary software, which gives the opportunity to analyze the image of microstructures, would allow to assess the ratio of phase structures and determine the ranges of changes in the phase composition better. The availability of these data significantly facilitates and supplements the studies.

\section{Conclusion}

Thus, the use of modern technologies and analysis of microstructure images can significantly speed up and simplify the research process. The study gives the opportunity to determine that carburization of surface, pre-hardened by wave deformation provides a more finely dispersed, even and densely-packed microstructure than hardening by wave deformation of previously carburized surface. This helps to improve the mechanical properties of the hardened surface and allows to provide for their smooth distribution over the whole section of the part.

\section{References}

[1] Kuts Yu.V., Povctyanoy A.Yu. Modern methods of microstructure research with the help of computer materials science using applied programs Naukovi Notatki, 2014, no.45, pp.323-329

[2] Kirichek A.V., Solovyev D.L., Khandozhko A.V., Fedonina S.O. Technological support of carrying layer parameters by deformation and combined strengthening. Science Intensive Technologies in Mechanical Engineering, 2018, no.10, vol. 88, pp. 43-48

[3] Kirichek A.V., Solovyev D.L., Silantyev S.A., Fedonina S.O. Influence of hardening by wave deformation on the material microstructure. Science Intensive Technologies in Mechanical Engineering, 2019, no.4, vol. 94, pp.13-17.

[4] Putyanin E.P. Image processing in robotics. Moscow. Mashinostroeniye, 1990. 320p.

[5] Chichko A.N., Sachek O.A., Likhuzov S.G. Software and algorithms for analyzing images of perlitic steel microstructures. Programmnye Produkty I Sistemy,2010, no.4, pp. 123-127

[6] M. Andersson, B. Holmquist, J. Lindquist, O. Nilsson, K.G. Wahlund, Analysis of film coating thickness and surface area of pharmaceutical pellets using fluorescence microscopy and image analysis, J. Pharm. Biomed. 22 (2000) P.325- 339
[7] Bodla KK, Murthy JY, Garimella SV. Microtomography-based simulation of transport through open-cell metal foams. Numer Heat Transfer Part A 2010;58(7):527.

[8] Mandelbrot B.B. The Fractal Geometry of Nature. N.Y.:Freeman.-San Francisco.-1982., P.351

[9] Stampfl J. Determination of the fracture toughness with automatic image processing / Stampfl J., S.Scherer, M.Gruber, O.Kolednik: Int. J. Frac., V.2-44, 1996 - P.139

[10] Montminy M.D., Tannenbaum A, MacOsko C.W. The 3D structure of real polymer foams. J Colloid Interface Sci 2004;280(1):202.

[11] L.A. Feldkamp, L.C. Davis, J.W. Kress, Practical cone beam algorithm, J. Microsc. 185 (1997) 67-75.

[12] Whitehouse D. Handbook of Surface Metrology // Institute of Physics Publishing, Bristol and Philadelphia, 1994. -988 p. 